\title{
COMMENTARY
}

\section{Serum adipocyte fatty acid-binding protein in the critically ill}

\author{
Undurti N Das*1-3 \\ See related research by Huang et al., http://ccforum.com/content/17/1/R22
}

\begin{abstract}
Sepsis due to unabated inflammation is common. Increased production of pro-inflammatory cytokines, free radicals, and eicosanoids has been detected in sepsis and other critical illnesses but could also be due to decreased synthesis and release of antiinflammatory molecules. Increased serum adipose-fatty acid-binding protein (A-FABP) levels can cause insulin resistance and have been reported in the critically ill, serve as a marker of prognosis, and thus link metabolic homeostasis and inflammation. A-FABP can be linked to the expression of Toll-like receptors, macrophage activation, synthesis and release of pro-inflammatory cytokines interleukin- 6 and tumor necrosis factoralpha, activation of cyclooxygenase 2 (COX-2) expression, and eicosanoid synthesis, events that can cause insulin resistance and initiation and progression of inflammation and sepsis. Unsaturated fatty acids and their anti-inflammatory products, such as lipoxins, resolvins, and protectins, may suppress A-FABP expression, inhibit macrophage and COX-2 activation, and decrease production of pro-inflammatory cytokines and ultimately could lead to a decrease in insulin resistance and resolution of inflammation and recovery from sepsis. Serial measurement of these pro- and anti-inflammatory molecules and correlation of their levels to the progression to or recovery from (or both) sepsis and other inflammatory processes may form a new approach to predict prognosis in inflammatory conditions and eventually could lead to the development of new therapeutic strategies.
\end{abstract}

\section{Introduction}

In a study in the previous issue of Critical Care, Huang and colleagues [1] observed that, in the critically ill,

*Correspondence: undurti@hotmail.com

'UND Life Sciences, 13800 Fairhill Road, \#321, Shaker Heights, OH 44120, USA

Full list of author information is available at the end of the article adipose-fatty acid-binding protein (A-FABP) concentrations were elevated and that the serum A-FABP concentrations were independently related to serum creatinine, fasting plasma glucose, total cholesterol, tumor necrosis factor-alpha (TNF- $\alpha$ ), albumin, and the Acute Physiology and Chronic Health Evaluation II scores, suggesting that higher A-FABP levels $(>40 \mathrm{ng} / \mathrm{mL}$ ) were associated with an unfavorable outcome in patients with sepsis. These results not only are interesting but also suggest that perhaps A-FABP could be used a biomarker of prognosis in the critically ill. But it is not clear why A-FABP levels should be increased in the critically ill or what this increase signifies.

Adipocyte fatty acid-binding protein (A-FABP or FABP4), also known as aP2 (adipocyte protein 2), is a carrier protein for fatty acids and is expressed primarily in adipocytes and macrophages. A-FABP belongs to the fatty acid-binding protein super-family whose members have relative molecular masses of approximately 15,000. A-FABP is a predominant cytosolic protein of mature adipocytes, accounts for approximately $6 \%$ of total cellular proteins, and is an important regulator of systemic insulin sensitivity and lipid and glucose metabolism [2]. Mice deficient in A-FABP are protected from development of hyperinsulinemia, hyperglycemia, and insulin resistance [3]. Adipocytes obtained from A-FABP-null mice had markedly reduced efficiency of lipolysis in vivo and in vitro [4] and showed a two- to three-fold decrease in fatty acid release, suggesting that A-FABP regulates efflux of fatty acids under normal physiological conditions. Acute insulin secretory response to $\beta$-adrenergic stimulation was profoundly suppressed in A-FABP ${ }^{-/-}$mice compared with their wild-type littermates [4], indicating that A-FABP could regulate systemic insulin sensitivity through its actions on other distal target tissues.

Adipose-fatty acid-binding protein and inflammation A-FABP is also present in macrophages, and its expression in macrophages can be induced by oxidized lowdensity lipoprotein (LDL) [5] and Toll-like receptor (TLR) agonists [6] and suppressed by statins [7]. A-FABP modulates inflammatory cytokine production and 


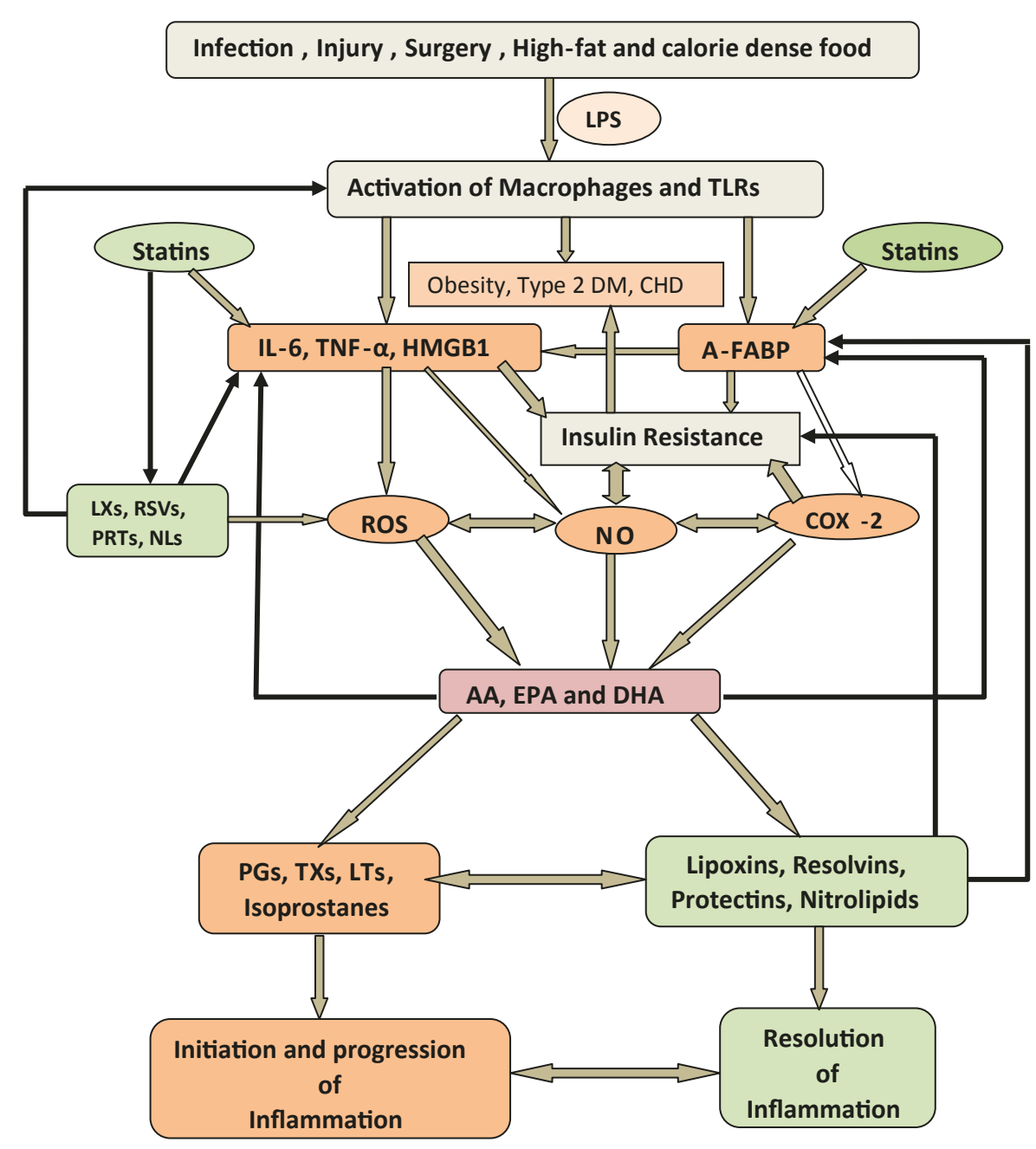

Figure 1. Interaction(s) among adipose-fatty acid-binding protein (A-FABP), Toll-like receptor (TLR), cytokines, free radicals, unsaturated fatty acids, and their products and inflammation and resolution of inflammation. Infections, injuries (including surgery), and high-fat diet activate macrophages and TLRs, leading to secretion of increased amounts of pro-inflammatory cytokines that, in turn, produce an excess of free radicals. Pro-inflammatory cytokines, TLRs, and free radicals activate A-FABP and cyclooxygenase 2 (COX-2), leading to increased production of pro-inflammatory prostaglandins (PGs), leukotrienes (LTs), and thromboxanes (TXs) from unsaturated fatty acids and decreases in the synthesis and release of anti-inflammatory lipoxins (LXs), resolvins (RSVs), and protectins (PRTs). Blocking the expression of A-FABP and TLRs will suppress inflammation. Unsaturated fatty acids and LXS, RSVS, and PRTs are expected to suppress macrophage activation and expression of A-FABP and TLRs and inhibit inflammation. Increased expression of A-FABP also occurs in obesity, type 2 diabetes mellitus (DM), and coronary heart disease (CHD). Interleukin-6 (IL-6), tumor necrosis factor-alpha (TNF-a), macrophage activation, increased expression of A-FABP, and increased production of PGs, LTs, TXs, and free radicals enhance insulin resistance in infections and sepsis. AA, arachidonic acid; DHA, docosahexaenoic acid; EPA, eicosapentaenoic acid; HMGB1, high-mobility group box 1; LPS, lipopolysaccharide; NL, nitrolipid; NO, nitric oxide; ROS, reactive oxygen species.

cholesterol ester accumulation [8]. Ablation of the $A-F A B P$ gene protected against atherosclerosis [9]. This evidence suggests that A-FABP, by integrating metabolic and inflammatory pathways, provides a key link between components of metabolic syndrome, implying that blocking A-FABP protein could be considered in the treatment of heart disease, diabetes mellitus, asthma, obesity, and fatty liver disease, which are all inflammatory conditions.

In this context, it is interesting to note that $\mathrm{A}-\mathrm{FABP}^{-/}$ mice are protected from experimental autoimmune encephalomyelitis and showed reduced levels of proinflammatory cytokine mRNA expression in central nervous system tissue as compared with wild-type mice. In vitro, antigen recall responses of myelin oligodendrocyte glycoprotein 35-55-immunized A-FABP ${ }^{-/-}$mice showed reduced proliferation and impaired interferongamma production. Dendritic cells deficient in FABPs were poor producers of pro-inflammatory cytokines interleukin-6 (IL-6) and TNF- $\alpha$ - and did not promote pro-inflammatory $\mathrm{T}$-cell responses, suggesting that metabolic-inflammatory pathway cross-regulation by 
A-FABPs plays a significant role in adaptive immune responses and inflammation [10]. These results - coupled with the observations that unsaturated fatty acids, such as palmitoleic acid, oleic acid, linoleic acid, linolenic acid, and eicosapentaenoic acid, significantly repressed the basal as well as lipopolysaccharide-induced A-FABP expression in macrophages and depletion of A-FABP expression by RNA interference (RNAi) decreased cyclooxygenase 2 (COX-2) mRNA expression and potentiated the repression by linoleic acid [11] - give interesting insights into the relationship among A-FABP, cytokines, and unsaturated fatty acids and their involvement in sepsis and other critical illnesses.

\section{Adipose-fatty acid-binding protein, Toll-like receptors, unsaturated fatty acids, and inflammation}

Recent studies [12,13] revived the controversy of whether unsaturated fatty acids are beneficial in the management of sepsis. The fact that A-FABP is increased in sepsis, induced by oxidized LDL and TLR agonists, and suppressed by statins and enhances the production of IL-6 and TNF- $\alpha$ and COX-2 expression whereas unsaturated fatty acids suppress $A-F A B P$ expression and IL- 6 and TNF- $\alpha$ production [14] indicates the complexity of the involvement of TLRs, A-FABP, cytokines, unsaturated fatty acids, and their products in sepsis.

The unsaturated fatty acid arachidonic acid (AA) is the precursor of two series of prostaglandins (PGs), two series of thromboxanes (TXs), and four series of leukotrienes (LTs); eicosapentaenoic acid (EPA) is the precursor of three series of PGs, three series of TXs, and five series of LTs. PGs, TXs, and LTs formed from EPA are less pro-inflammatory in nature compared with PGs, TXs, and LTs formed from AA but still are proinflammatory in nature [15]. Interestingly, AA, EPA, and docosahexaenoic acid (DHA) suppress the production of pro-inflammatory cytokines IL-6 and TNF- $\alpha$ both in vitro and in vivo. In addition, free radicals act on unsaturated fatty acids to give rise to F2-isoprostanes, a group of pro-inflammatory substances, whereas nitric oxide and unsaturated fatty acids interact to form nitrolipids that have anti-inflammatory action [15].

Furthermore, AA is the precursor of lipoxin $\mathrm{A}_{4}\left(\mathrm{LXA}_{4}\right)$, and EPA and DHA give rise to resolvins and DHA gives rise to protectins; resolvins and protectins have potent anti-inflammatory actions, resolve inflammation, and enhance wound healing by suppressing the production of free radicals, myeloperoxidase, IL-6, TNF- $\alpha$, and HMGB1 (high-mobility box 1) and antagonize the pro-inflammatory actions of leukotrienes [15]. Resolvins and protectins block TLR-mediated activation of macrophages [15] and enhance the anti-bacterial action of antibiotics and augment the clearance of bacteria [16]. This implies that the optimal production of lipoxins, resolvins, and protectins from unsaturated fatty acids is essential for clearing the invading pathogens, resolving inflammation, and preventing tissue damage.

\section{Balance between pro- and anti-inflammatory events and molecules determines prognosis}

Under normal physiological conditions, a balance between pro- and anti-inflammatory molecules is maintained. A tilting of this balance in favor of the former would lead to initiation and progression of inflammation, insulin resistance, and consequent tissue damage [17]. Such an inflammatory process could be due not only to increased expression of $A-F A B P$ and augmented production of proinflammatory molecules but also to deficiency of antiinflammatory molecules such as IL-4, IL-10, lipoxins, resolvins, and protectins (Figure 1). In this context, it is noteworthy that A-FABP suppresses cardiomyocyte contraction [18] whereas unsaturated fatty acids seem to prevent cardiac failure [19], reinforcing close interaction(s) among various pro- and anti-inflammatory molecules and their role in sepsis. Hence, a better understanding of the interaction(s) among these molecules in inflammatory conditions is essential in order to develop meaningful and novel therapeutic strategies especially for sepsis.

\section{Abbreviations}

AA, arachidonic acid; A-FABP, adipose-fatty acid-binding protein; COX-2, cyclooxygenase 2; DHA, docosahexaenoic acid; EPA, eicosapentaenoic acid; IL, interleukin; LDL, low-density lipoprotein; LT, leukotriene; PG, prostaglandin; TLR, Toll-like receptor; TNF-a, tumor necrosis factor-alpha; TX, thromboxane.

Competing interests

The author declares that he has no competing interests.

\section{Acknowledgments}

UND is the recipient of a Ramalingaswami Fellowship from the Department of Biotechnology, New Delhi, India. This work was supported by grants from the Department of Biotechnology (BT/PR1 1627/MED/30/157/2010), the Department of Science and Technology (IR/SO/LU/03/2008/1) under Intensification of Research in High Priority Areas (IRPHA), and the Defence Research and Development Organisation, New Delhi (TC/2519/INM-03/2011/ CARS) under R\&D Project INM-311.

\section{Author details}

'UND Life Sciences, 13800 Fairhill Road, \#321, Shaker Heights, OH 44120, USA. Bio-Science Research Centre, Gayatri Vidya Parishad College of Engineering, Visakhapatnam-533 048, India. ${ }^{3}$ Jawaharlal Nehru Technological University, Kakinada-533003, India.

\section{Published: 6 March 2013}

\section{References}

1. Huang C-L, Wu Y-W, Hsieh A-R, Hung Y-H, Chen W-J, Yang W-S: Serum adipocyte fatty acid-binding protein levels in critical illness patients are associated with insulin resistance and predict mortality. Crit Care 2013, 17:R22.

2. Makowski L, Hotamisligil GS: Fatty acid binding proteins - the evolutionary crossroads of inflammatory and metabolic responses. J Nutr 2004 134:2464S-2468S.

3. Uysal KT, Scheja L, Wiesbrock SM, Bonner-Weir S, Hotamisligil GS: Improved glucose and lipid metabolism in genetically obese mice lacking aP2. Endocrinology 2000, 141:3388-3396. 
4. Coe NR, Simpson MA, Bernlohr DA: Targeted disruption of the adipocyte lipid-binding protein (aP2 protein) gene impairs fat cell lipolysis and increases cellular fatty acid levels. J Lipid Res 1999, 40:967-972.

5. Fu Y, Luo N, Lopes-Virella MF: Oxidized LDL induces the expression of ALBP/ aP2 mRNA and protein in human THP-1 macrophages. J Lipid Res 2000, 41:2017-2023.

6. Kazemi MR, McDonald CM, Shigenaga JK, Grunfeld C, Feingold KR: Adipocyte fatty acid-binding protein expression and lipid accumulation are increased during activation of murine macrophages by toll-like receptor agonists. Arterioscler Thromb Vasc Biol 2005, 25:1220-1224.

7. Llaverias G, Noé V, Peñuelas S, Vázquez-Carrera M, Sánchez RM, Laguna JC, Ciudad CJ, Alegret M: Atorvastatin reduces CD68, FABP4, and HBP expression in oxLDL-treated human macrophages. Biochem Biophys Res Commun 2004, 318:265-274.

8. Makowski L, Brittingham KC, Reynolds JM, Suttles J, Hotamisligil GS: The fatty acid-binding protein, aP2, coordinates macrophage cholesterol trafficking and inflammatory activity. Macrophage expression of aP2 impacts peroxisome proliferator-activated receptor $\gamma$ and IKB kinase activities. J Biol Chem 2005, 280:12888-12895.

9. Boord JB, Maeda K, Makowski L, Babaev VR, Fazio S, Linton MF, Hotamisligil GS: Combined adipocyte-macrophage fatty acid-binding protein deficiency improves metabolism, atherosclerosis, and survival in apolipoprotein E-deficient mice. Circulation 2004, 110:1492-1498.

10. Reynolds JM, Liu Q, Brittingham KC, Liu Y, Gruenthal M, Gorgun CZ, Hotamisligil GS, Stout RD, Suttles J: Deficiency of fatty acid-binding proteins in mice confers protection from development of experimental autoimmune encephalomyelitis. J Immunol 2007, 179:313-321.

11. Coleman SL, Park YK, Lee JY: Unsaturated fatty acids repress the expression of adipocyte fatty acid binding protein via the modulation of histone deacetylation in RAW 264.7 macrophages. Eur J Nutr 2011, 50:323-330.

12. Pradelli L, Mayer K, Muscaritoli M, Heller AR: $\mathrm{n}-3$ fatty acid-enriched parenteral nutrition regimens in elective surgical and ICU patients: a meta-analysis. Crit Care 2012, 16:R184.
13. Gadek JE, DeMichele SJ, Karlstad MD, Pacht ER, Donahoe M, Albertson TE, Van Hoozen C, Wennberg AK, Nelson JL, Noursalehi M: Effect of enteral feeding with eicosapentaenoic acid, gamma-linolenic acid, and antioxidants in patients with acute respiratory distress syndrome. Enteral Nutrition in ARDS Study Group. Crit Care Med 1999, 27:1409-1420.

14. Kumar SG, Das UN: Effect of prostaglandins and their precursors on the proliferation of human lymphocytes and their secretion of tumor necrosis factor and various interleukins. Prostaglandins Leukot Essen Fatty Acids 1994, 50:331-334.

15. Das UN: Molecular Basis of Health and Disease. New York: Springer; 2011.

16. Chiang N, Fredman G, Bached F, Oh SF, Vickery T, Schmidt BA, Serhan CN: Infection regulates pro-resolving mediators that lower antibiotic requirements. Nature 2012, 484:524-529.

17. Das UN: Is sepsis a pro-resolution deficiency disorder? Med Hypotheses 2013, 80:297-299.

18. Lamounier-Zepter V, Look C, Alvarez J, Christ T, Ravens U, Schunck WH, Ehrhart-Bornstein M, Bornstein SR, Morano I: Adipocyte fatty acid-binding protein suppresses cardiomyocyte contraction: a new link between obesity and heart disease. Circ Res 2009, 105:326-334.

19. Hara M, Sakata Y, Nakatani D, Suna S, Usami M, Matsumoto S, Hamasaki T, Do Y, Nishino M, Sato H, Kitamura T, Nanto S, Hori M, Komuro I, for the Osaka Acute Coronary Insufficiency Study (OACIS) Investigators: Low levels of serum $n-3$ polyunsaturated fatty acids are associated with worse heart failure-free survival in patients after acute myocardial infarction. Circ 2013, 77:153-162.

doi:10.1186/cc12517

Cite this article as: Das UN: Serum adipocyte fatty acid-binding protein in the critically ill. Critical Care 2013, 17:121. 